\title{
Mercury-Waterfilling for Generalized Multicarrier Signaling
}

\author{
Adrian Kliks
}

Published online: 13 March 2013

(C) The Author(s) 2013. This article is published with open access at Springerlink.com

\begin{abstract}
Orthogonal Frequency Division Multiplexing is often treated as the promising technique for next wireless or wired communication systems. Beside its great advantages, orthogonal frequency division multiplexing has also some weaknesses, such as high out-ofband emission or high peak-to-average power ratio, which become the basis for investigation on new transmission techniques. In this paper the application of the so-called generalized multicarrier (GMC) signaling is considered as a good solution for application in future systems, especially for cognitive-radio applications. It is characterized by high flexibility and adaptability of its parameters, thus allowing for e.g. low out-of-band emission. In this work the possibilities of application of link adaptation techniques have been analyzed, with the particular attention put on the mercury-waterfilling principle. Two approaches of application of this procedure in GMC systems have been proposed and analyzed by means of computer simulations.
\end{abstract}

Keywords Cognitive radio $\cdot$ Multicarrier transmission $\cdot$ Link adaptation · Mercury and waterfilling

\section{Introduction}

Multicarrier signals have been already considered for application in many wireless and wired communication systems, just to mention IEEE 802.11 [1], IEEE 802.16 [2] or DVB-T/H standards [3], as well as the so-called 4G cellular systems. Currently the most popular approach in practical realizations of multicarrier systems is to make the parallel subcarriers orthogonal to each other. Such an approach leads to the well-known concept of the Orthogonal Frequency Division Multiplexing (OFDM) [4], whose advantages are compelling and undisputed. The possibility of signal's parameter adaptation to the current channel realization, efficient hardware realization (due to e.g. dedicated chips calculating fast Fourier transforms-FFT) as

A. Kliks $(\varangle)$

Chair of Wireless Communications, Poznan University of Technology, Polanka 3, 60-965 Poznan, Poland

e-mail: akliks@et.put.poznan.pl 
well as simplicity of channel estimation (high signal robustness on multipath interference) explain high popularity of the OFDM technique. Nevertheless, OFDM transmission has also some drawbacks that become often the rationale for further investigation on new multicarrier transmission schemes. Recently, two approaches have gained lot of attention, i.e. FilterBank based MultiCarrier (FBMC) modulation and Non-Orthogonal Frequency Division Multiplexing (NOFDM) [5-13]. In both schemes the consecutive user data symbols are transmitted on specific subcarriers, appropriately filtered in order to fulfill some predefined requirements. In both cases the usage of the so-called cyclic prefix, as it is known in OFDM-based systems, is not mandatory. Moreover, the transmission is realized in the form of the time-frequency frames, i.e. one transmission frame consists of $L \times M$ pulses on the Time-Frequency (TF) plane. It further means that $L$ consecutive blocks of $M$ parallel pulses are transmitted in one FBMC/NOFDM frame. Moreover, one of the key aspects of the FBMC/NOFDM-based system design is the proper definition of the transmit and the receive pulse shapes (filters), denoted as $g(t)$ and $q(t)$, respectively. Various pulse-shapes have been derived for FBMC in the rich literature, such as Isotropic Orthogonal Transform Algorithm pulse (IOTA) [14], Hermitian, Enhanced Gaussian Function (EGF) pulse [11], etc. Although these pulses are often designed in such a way that the orthogonality is ensured, one of the drawbacks of the practical implementation of the FBMC/NOFDM-based system in practice is the presence of the residual self-interference due to the impairments of electronic elements, finite representation of the pulses etc. This effect has to be considered during analysis of potential application of link adaptation techniques, since the presence of residual interference influences the values of observed Signal-to-Interference-plus-Noise ratio (SINR), what entails e.g. different bit and power allocation as it will be for OFDM systems.

It can be observed that all of the assumptions made in the orthogonal (e.g. OFDM) and non-orthogonal systems (e.g. NOFDM) or multicarrier systems realized by means of filterbanks (FBMC) can be generalized and included in the broader concept of the Generalized MultiCarrier (GMC) transmission $[15,16]$. In such an approach, the shapes of the transmit and receive pulses are not pre-defined and can be theoretically modified in order to fulfill some specific requirements. Moreover, the elements of the set transmit pulses (used at the transmitter side) and the set of receive pulses (used at the receiver) do not have to be mutually orthogonal. The only one requirement put in front of the GMC signaling is that in order to allow seamless transmission these two sets of pulses have to fulfill some specific criterion or constraint. But this criterion can be also defined freely. In case of FBMC/NOFDM systems, the biorthogonality of any pair of elements taken from the sets of transmit and receive pulses has to be ensured, while in case of OFDM, these pulses have to be additionally mutually orthogonal. In case of GMC transmission the distance between the neighboring pulses in time and frequency domain are also parametrized and can be selected freely. In the context of cognitive radio the pair of transmit and receive pulses will be defined in such a way such that the amount of power emitted outer the nominal transmission band will be minimized. Since GMC is the broader class of signals than OFDM one, most of the algorithms developed for OFDM or even FBMC cannot be applied in the straight-forward manner for GMC signals. Some specific features of GMC systems have to be taken into account, e.g. the phenomena of possibly strong overlapping of neighboring pulses on TF plane. In this paper the idea of application of the Mercury-Water Filling Principle (MFP) [17-20] in GMC systems is considered. The rationale of the mercury-filling concept, originally proposed for OFDM systems, is the observation that WaterFilling Principle (WFP) [21], known as the best power allocation strategy for multicarrier systems, is optimal only under the assumption that the input values to the channel are Gaussian distributed. This is however generally not true, since in most communication systems various constellation of symbols (e.g. QAM symbols) are used. The 
authors of $[17,18,20]$ have showed the benefits that one can gain from possessing of the knowledge about the character of input data. But, as stated above, the mercury-waterfilling concept have been developed for OFDM systems. In this work the MFP has been applied for the GMC systems.

The reminder of the paper is organized as follows. First, in Sect. 2, the concept of the GMC systems will be analyzed in a more detailed way. In Sect. 3 the basics of the mercuryfilling algorithm will be discussed, followed by the presentations of two approaches, how this principle can be applied in the case of GMC systems. Finally, Sect. 4 treats the simulations results, while Sect. 5 summarizes and concludes the work.

\section{The Concept of Generalized Multicarrier Signaling}

When focusing on the OFDM systems one can state that in order to ensure orthogonality between the adjacent subcarriers the set of specific requirements has to be fulfilled, e.g. the shape and the time-support of the transmit pulse $g(t)$ are strictly defined. In most practical applications the shape of the transmit pulse, but also of the receive pulse (denoted hereafter as $q(t)$ ), is rectangular, or including filtering-a modification of (square-)root-raised-cosine one, and its duration $T$ is strictly related to the distance between the neighboring subcarriers in frequency domain $F$, i.e. the following relation holds $F=\frac{1}{T}$. Beside its great potential, OFDM signals suffers from some significant problems, such us the need of cyclic-prefix addition, high sensitivity to the frequency synchronization errors and high amplitude variations of the time-domain signal. These observations become the rationale of founding new ways of data transmission, and recently - as already stated-FBMC/NOFDM techniques have gained lot of interest. In this approach there is no need for addition of cyclic prefix to the transmitted signal. Moreover, some rules regarding the shape of the transmit and receive pulses have been relaxed, i.e. slight overlapping of neighboring pulses has been allowed. These two approaches can be generalized and represented as the special cases of the so-called GMC signals. In that case neither the density of pulse allocation on the TF plane nor the pulse shape are usually specified $[12,13,22,23]$. The distance between the pulses on the TF plane and the transmit pulse shape can be theoretically chosen without any constraints. The presence of the cyclic prefix is also not obligatory. As in OFDM or FMBC cases, the GMC signaling assumes the usage of transmit frames, i.e. user data is carried by the pulses organized in form of a frame on TF plane. The exemplary GMC transmission frame that consists of $N_{\text {tot }}$ pulses divided into $L$ blocks of $M$ shifted in frequency pulses is showed in Fig. 1. In that figure one ellipse illustrates the TF representation of the applied transmit pulse. Keeping the notation introduced for the OFDM signals, the time-distance (expressed in the assumed time-units or samples depending on the considered system) between the consecutive pulses is equal to $T_{S}$, the frequency distance (subcarrier spacing) is denoted by $F$, while time-support is represented by $T$. One can observe that the adjacent pulses on the TF plane overlap each other causing the so-called self-interference.

In order to recover the transmit data at the receiver, the shape of the receive filter $q(t)$ has to be appropriately defined in order to mitigate the lack of orthogonality between the transmit pulses. By the term "appropriately" we mean the way in which some predefined criterion will be fulfilled. Usually, the biorthogonality criterion has to be ensured, i.e. every element from the set of translated (shifted in time) and modulated (shifted in frequency) functions $g(t)$ is orthogonal to each translated and modulated function $q(t)$ used at the receiver. Based on the aforementioned analysis, the generic multicarrier signal (i.e. one frame that consists of $L$ blocks of $M$ pulses) can be represented in discrete form as follows: 
Fig. 1 Exemplary GMC frame

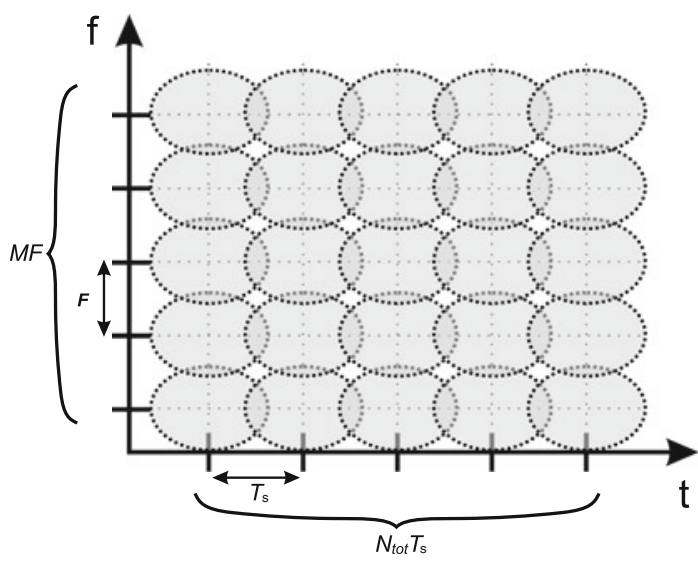

$$
s[k]=\sum_{l=0}^{L-1} \sum_{m=0}^{M-1} d_{l, m} \cdot g[k-l N] \cdot e^{2 j \pi k m / M},
$$

where $N$ is the distance (in samples) between adjacent pulses in time domain, $d_{l, m}$ defines the user data (e.g. QAM symbols) transmitted on the $m$-th subchannel in the $l$-th time slot in one frame and $g[k]$ defines the so-called synthesis window (or transmit pulse). All transmit pulses (shifted both in time and in frequency) constitutes the set basis functions $G=\left\{g_{l, m}[k]=\right.$ $\left.g[k-l N] \cdot e^{2 j \pi k m / M}\right\}$ used at the transmitter for generation of the time domain signal. At the receiver the set of biorthogonal basis functions, i.e. $Q=\left\{q_{l, m}[k]=q[k-l N] \cdot e^{2 j \pi k m / M}\right\}$, has to be used. As already mentioned, in oder to recover the transmit data perfectly, the biorthogonal criterion has to be fulfilled, i.e. $\sum_{l=0}^{L} \sum_{m=0}^{M-1} g_{l, m}[k] q_{l, m}\left[k^{\prime}\right]=\delta\left[k-k^{\prime}\right]$, where $\delta[\cdot]$ is the Kronecker delta function [13]. Furthermore, the received signal (in discrete form) can be defined as follows:

$$
y[n]=\sum_{l=0}^{L-1} \sum_{m=0}^{M-1} h_{l, m} d_{l, m} g_{l, m}[n]+\eta_{l, m}[n],
$$

where $h_{l, m}$ denotes the channel coefficient, and $\eta_{l, m}$ is the AWGN sample observed at the $(l, m)$ location of TF plane. When focusing on the specific pulse located at the certain TF point, and taking into account that the neighboring pulses overlap in time and frequency domains and the self-interference cannot be in practice eliminated completely, the received signal can be represented as in (3).

$$
\begin{aligned}
y_{l, m}[n] & =\sum_{l=0}^{L-1} \sum_{m=0}^{M-1} h_{l, m} p_{l, m} d_{l, m} g_{l, m}[n]+\eta_{l, m}[n] \\
& =\overbrace{h_{l, m} p_{l, m} d_{l, m} g_{l, m}[n]}^{\text {Desiredpart }}+\overbrace{\sum_{\left.l^{\prime}, m^{\prime}\right) \in \mathbb{D}_{l, m}} h_{l^{\prime}, m^{\prime}} p_{l^{\prime}, m^{\prime}} d_{l^{\prime}, m^{\prime}} g_{l^{\prime}, m^{\prime}}[n]}^{\text {Potentialharmfulpart(self-interference) }}+\overbrace{\eta_{l, m}[n]}^{\text {Noise }}
\end{aligned}
$$

In such an approach the desired part, as well as unwanted components have been distinguished. Moreover, one can find the estimate of the user data $\tilde{d}_{l, m}$ carried by that pulse obtained at the receiver after the filtering process by the corresponding filter $q_{l, m}$ as presented in (4), where $\Delta \epsilon_{l^{\prime}, m^{\prime}}^{(l, m)}=\sum_{n} g_{l, m} q_{l^{\prime}, m^{\prime}}^{*}$ expresses the value of the inner product of the 
transmit pulse $g_{l, m}$ localized on the $(l, m)$ point on the TF plane and the receive pulse $q_{l^{\prime}, m^{\prime}}$ localized on the $\left(l^{\prime}, m^{\prime}\right)$ point on the TF plane. In the ideal case $\Delta \epsilon_{l, m}^{(l, m)}=1$ and $\Delta \epsilon_{l^{\prime}, m^{\prime}}^{(l, m)}=0$.

$$
\begin{aligned}
\tilde{d}_{l, m}= & \sum_{n}\left(\sum_{l=0}^{L-1} \sum_{m=0}^{M-1} h_{l, m} p_{l, m} d_{l, m} g_{l, m}[n]+\eta_{l, m}[n]\right) q_{l, m}^{*}[n] \\
= & \overbrace{h_{l, m} p_{l, m} d_{l, m} \sum_{n} g_{l, m}[n] \cdot q_{l, m}^{*}[n]}^{\text {Desiredpart }} \\
& +\overbrace{\sum_{\left(l^{\prime}, m^{\prime}\right) \in \mathbb{D}_{l, m}} h_{l^{\prime}, m^{\prime}} p_{l^{\prime}, m^{\prime}} d_{l^{\prime}, m^{\prime}} \sum_{n} g_{l^{\prime}, m^{\prime}}[n] \cdot q_{l, m}^{*}[n]}^{\text {Residualself-interference }}+\overbrace{\sum_{n} \eta_{l, m}[n] \cdot q_{l, m}^{*}[n]}^{\text {Colorednoise }} \\
= & h_{l, m} d_{l, m} p_{l, m} \cdot \Delta \epsilon_{l, m}^{(l, m)} \\
& +\sum_{\left(l^{\prime}, m^{\prime}\right) \in \mathbb{D}_{l, m}} h_{l^{\prime}, m^{\prime}} d_{l^{\prime}, m^{\prime}} p_{l^{\prime}, m^{\prime}} \Delta \epsilon_{l^{\prime}, m^{\prime}}^{(l, m)}+\sum_{n} \eta_{l, m}[n] \cdot q_{l, m}^{*}[n]
\end{aligned}
$$

However, in practice $\Delta \epsilon_{l, m}^{(l, m)} \approx 1$ and $\Delta \epsilon_{l^{\prime}, m^{\prime}}^{(l, m)} \approx 0$. It is mainly due to the fact that pair of transmit and receive pulses cannot be derived in such a way that the predefined constraints (as biorthogonality) is fulfilled ideally, e.g. the duration of the pulses has to be finite. Thus, one can observe the presence of the unwanted part of the signal, i.e. the residual self-interference resulting from the unideal design of the pair of transmit and receive pulses $(g(t), q(t))$. The second term in (3) and (4) represents the total amount of interference introduced from the surrounding pulses to the pulse localized on the $(l, m)$ point on the TF plane. The pairs of indexes of the surrounding pulses in one GMC frame belongs to the set $\mathbb{D}_{l, m}$, defined as $\mathbb{D}_{l, m}=\left\{\left(l^{\prime}, m^{\prime}\right) \in \mathbb{Z}^{2}:\left(l^{\prime}, m^{\prime}\right) \neq(l, m) \wedge\left(0 \leq l^{\prime} \leq L-1\right) \wedge\left(0 \leq m^{\prime} \leq M-1\right)\right\}$. Finally, the equivalent noise component is defined as $\tilde{\eta}_{l, m}=\sum_{n} \eta_{l, m}[n] \cdot q_{l, m}^{*}[n]$.

\subsection{Projection of OFDM Signals on GMC Signal Space}

For completeness of the analysis let us project the OFDM onto the space of GMC signals. In a case of OFDM transmission both pulses are identical (rectangular), i.e. $g[k]=q[k]$, and the above biorthogonality condition reduces to the orthogonality one. Moreover, duration of both pulses (without cyclic prefix) is equal to distance between the two consecutive pulses in time domain $T_{\mathrm{s}}=T$, and the subcarrier spacing is equal to the reciprocity of the orthogonalization time. If the cyclic prefix of the duration $T_{\mathrm{cp}}$ is added, the distance between two pulses in time domain increases, i.e. $T_{\mathrm{s}}=T+T_{\mathrm{cp}}$. In typical systems the transmit frames are of size $L \times M=1 \times M$ pulses on TF plane. Finally, due to the simplicity of transmit and receive filter definition, the level of remaining residual self-interference is small, assuming that the proper time and frequency synchronization is ensured. In that light, other signals (e.g. FBMC, NOFDM, even single carrier) can be also mapped on the GMC class.

\subsection{Practical Realization}

One of the main advantages of OFDM-based systems is that the OFDM modulation can be efficiently realized by means of dedicated chips responsible for forward and inverse fast Fourier transformation. Undoubtedly, introduction of high flexibility in the definition of the transmit signal (various pulse shapes, parametrized distance between the pulses on TF plane 
etc.) in GMC systems entails the increase of overall complexity. Hopefully, GMC transceivers can be realized by the (I)FFT block followed by the bank of special polyphase filters [13,24]. Such an approach has been proposed already in the Filter-Bank based MultiCarrier systems and it has been shown that the increase of number of required operation per one transmit signal is relatively slight [15].

\subsection{Advantages of GMC Signaling in the Context of Cognitive Radio}

The GMC signaling has various properties that could be widely exploited especially in the context of the cognitive systems, where the unlicensed users can operate within the presence of primary, licensed users. First, let us observe the by using GMC representation one can define each kind of transmit signal by means of set of parameters, i.e. the OFDM signal will be defined by proper definition of the transmit and receive pulse shapes, their duration, length of cyclic prefix etc. In other words, each of the existing standards can be implemented when using GMC description. On the other hand, by application of the GMC one can define any shape of the transmit pulse depending on the current channel characteristics and available context information about the surroundings; the final form of the transmit signal can be also defined without any constraints (number and density of the pulses within one transmit frame on TF plane, etc.). For example, the secondary user can select such shape of the transmit pulse which will minimize the out-of-band power radiation (thus minimizing the interference introduced to the primary user) and this will allow the secondary user to start transmission even in the very narrow vacant frequency gap.

\subsection{Calculation of the Dual Pulse}

As it was mentioned in the previous section, in order to recover user data from the transmit signal (represented by means of frame $\left\{g_{l, m}\right\}$ ) one has to use the dual frame $\left\{q_{l, m}\right\}$. The assumption of the perfect recovery of coefficients $d_{l, m}$ in the case of non-dispersive and noise-free channel $[12,24]$ is fulfilled when the dual pulse prototype satisfies e.g. the biorthogonality condition. In such a case a problem of efficient calculation of the dual pulse arises. Various solutions have been proposed in the literature, e,g. the so-called straightforward method (derived from the biorthogonality criterion, [13]) which is rather complicated and computationally complex, or the fast algorithms proposed in [25-27]. It has to be mentioned that several procedures of calculation of the dual pulse have been proposed that are dedicated to specific applications, e.g. [28,29]. In this work, the algorithms proposed by Prinz [25] has been used.

\section{Application of the Mercury/Waterfilling Principle in GMC Scheme}

\subsection{Mercury-Filling: Basics}

Multicarrier signals consists of lower or higher number $M$ of parallel subcarriers. When applying this kind of signals one can try to benefits from the variations of the transmission channel, i.e. it is possible to adapt signal parameters according to the current status of the transmission channel. It is evident that the allocation of power as well as bits among available subcarriers should depend on the quality of the transmission channel. Intuitively, the better the channel for a certain subcarrier, the higher the number of bits (and thus power) assigned to this frequency bin. Various bit and power loading procedures, as well as approaches consid- 
ering application of modulation and coding schemes (MCS), have been proposed in the rich literature. Just to mention the solutions proposed by Hughes-Hartogs [30], Campello [31] or by Fisher and Huber [32]. All of these algorithms tends to reach the optimal, upper limit defined by the well-known water-filling principle (WFP), i.e. the power allocation among subcarriers maximizing the channel capacity [21]. However, the optimality of this approach is conditioned by the assumption that the input data are Gaussian distributed, what in typical communication systems is not valid. It has been showed $[17,18,20]$ that inclusion of this fact leads to the so-called mercury-filling principle (MFP). Let us analyze the properties of the MFP in the context of GMC transmission, in which the transmit frame consists of $N_{\text {tot }}=$ $M \times L$ pulses on TF plane. Denoting by $D=\left\{d_{l, m}: 0 \leq m \leq M-1 \wedge 0 \leq l \leq L-1\right\}$ the set of input symbols, the input-output mutual information for the $(m, l)$-th pulse is defined as:

$$
\mathcal{J}_{l, m}(\rho)=I\left(d_{l, m} ; \sqrt{\rho} d_{l, m}+\eta_{l, m}\right),
$$

where $\rho$ is the channel gain. Similarly, the estimate $\hat{d}_{l, m}$ of the transmitted symbol can be calculated as:

$$
\hat{d}_{l, m}\left(y_{l, m}, \rho\right)=E\left\{d_{l, m} \mid y_{l, m}=\sqrt{\rho} d_{l, m}+\eta_{l, m}\right\} .
$$

and the corresponding minimum mean squared error (MMSE) can be expressed as:

$$
\operatorname{MMSE}_{l, m}(\rho)=E\left(\left|d_{l, m}-\hat{d}_{l, m}\left(\sqrt{\rho} d_{l, m}+\eta_{l, m}, \rho\right)\right|^{2}\right),
$$

where $E(\cdot)$ denotes expectation. The basis of the aforementioned MFP is the observation that there exist straight-forward relation between the input-output mutual information (MI) $I(\gamma)$ and the MMSE function at the receiver $[17,18]$ :

$$
\frac{d}{d \gamma} I_{l, m}(\gamma)=\frac{1}{2} \operatorname{MMSE}_{l, m}(\gamma),
$$

where for AWGN channel $\gamma=\frac{P}{\sigma^{2}}$ is ratio between the signal power $P$ and the variance $\sigma^{2}$ of the noise observed at the location $(m, l)$-th pulse. Denoting the fraction of total power $P$ assigned to the $(m, l)$-th pulse by $p_{l, m}$, the signal-to-noise ratio (SNR) for the fading channel can be defined as $\gamma_{l, m}=\frac{p_{l, m} P\left|h_{l, m}\right|^{2}}{\sigma^{2}}$. In the following we assume that the total transmit power is constant and can be normalized, i.e. $P=P^{*}(=1)$, thus

$$
\sum_{l, m} p_{l, m}^{*} \leq 1
$$

In such a case the values of powers $\left\{p_{l, m}^{*}\right\}$ that maximize the sum mutual information can be calculated as presented in the formula below:

$$
\begin{aligned}
p_{l, m}^{*}=0 \text { for } \quad \gamma_{l, m}<\zeta \\
\gamma_{l, m} \cdot \operatorname{MMSE}_{l, m}\left(p_{l, m}^{*} \gamma_{l, m}\right)=\zeta \text { for } \quad \gamma_{l, m} \geq \zeta
\end{aligned}
$$

where $\zeta$ is found to fulfill the relation (9). Moreover, relation (10) can be expressed also in the conciser form:

$$
p_{l, m}^{*}=\frac{1}{\gamma_{m}} \operatorname{MMSE}_{m}^{-1}\left(\min \left(1, \frac{\zeta}{\gamma_{l, m}}\right)\right),
$$

for $\zeta$ being now the solution of one of the following formulas: 
1. if the power is constrained by $P^{*}$ :

$$
\sum_{(l, m) \wedge \gamma_{l, m}>\zeta} \frac{1}{M \gamma_{l, m}} \operatorname{MMSE}_{l, m}^{-1}\left(\frac{\zeta}{\gamma_{l, m}}\right)=P^{*} .
$$

2. if the total mutual information is constrained by $I^{*}$

$$
\frac{1}{M} \sum_{(l, m) \wedge \gamma_{l, m}>\zeta} I\left(\operatorname{MMSE}_{l, m}^{-1}\left(\frac{\zeta}{\gamma_{l, m}}\right)\right)=I^{*} .
$$

For a given $R$-ary modulation, consisting of the set of symbols $\mathcal{D}_{R}=\left\{d_{r}\right\}_{r=0}^{R-1}$, the MMSE can be represented in the closed form as defined in e.g. [18]. For low values of SINR $\gamma_{l, m}$, the MMSE function can be approximated as:

$$
\operatorname{MMSE}\left(\gamma_{a, b}\right)=1+I \ddot{(0)} \gamma_{a, b}+O\left(\gamma_{a, b}^{2}\right),
$$

where $I \ddot{(0)}$ is the value of the first derivative of the function $I(\cdot)$ in the point 0 , while $O(\cdot)$ is the upper bounded function (as in the Landau notation). For example, for low SINR values and for BPSK the formula (14) can be simplified, since $I \ddot{(0)}=-2$, thus the power allocation policy can be described as in (15):

$$
\forall_{(l, m)} \quad p_{l, m}=\frac{\max \left(0 ; \gamma_{l, m}-\zeta\right)}{2 \gamma_{l, m}^{2}} .
$$

\subsection{First Proposal}

In the first case let us try to apply the water-filling as well as mercury-filling principles in the straight-forward manner, i.e. we will concentrate on the transmit side and take into account the channel characteristics (assumed to be known at the transmitter side). In the original WFP and MFP the final power allocation among the subcarriers depends on the signal-to-interference noise ratio (SINR). As it was mentioned in the previous sections, in the case of GMC signals the transmit pulses can overlap even significantly on the TF plane. In practice, however, one can assume that this phenomena will be rather small, but not so small that it could be omitted. Thus, we propose to calculate the level of the interference observed by the pulse located at the point $(l, m)$ on TF plane and coming from the neighboring ones. Assuming that the power carried by one pulse is normalized, let us denote the power distribution on the TF plane of the transmit pulse $g_{l, m}(t)$ as $\Theta_{l, m}$. In such a case the new SINR value can be calculated as follows:

$$
\gamma_{l, m}=\frac{\Theta_{l, m}^{(l, m)}\left|h_{l, m}\right|^{2}}{\breve{I}_{l, m}+\sigma_{l, m}^{2}},
$$

where $\breve{I}_{l, m}$ is the power of interference observed by the pulse located at the point $(l, m)$ on TF plane and is defined as:

$$
\breve{I}_{l, m}=\sum_{\left(l^{\prime}, m^{\prime}\right) \in \mathbb{D}_{l, m}}\left|h_{l^{\prime}, m^{\prime}}\right|^{2} \Theta_{l^{\prime}, m^{\prime}}^{(l, m)} .
$$

In both above formulas the term $\Theta_{c, d}^{(a, b)}$ defines the amount of power originating from the pulse $g_{c, d}(t)$ observed at the point $(a, b)$ on the TF plane, or in other words, the power of interference introduced by the pulse localized at point $(c, d)$ into the pulse localized the point $(a, b)$. Let us stress that finding of the exact power distribution on the TF plane is a 

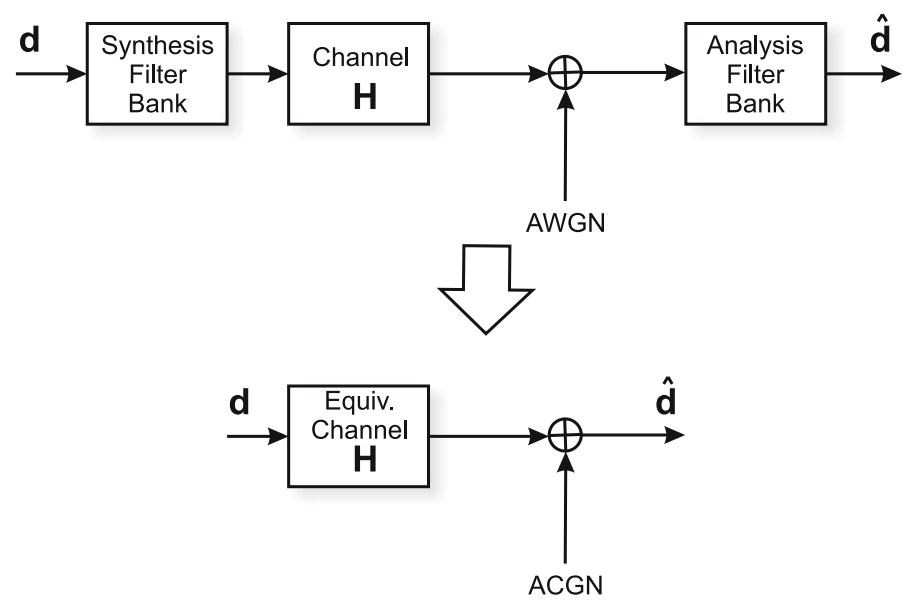

Fig. 2 Illustration of the idea of the equivalent channel model

separate research topic, since due to the Heisenberg uncertainty rule, the infinitely precise power distribution on TF plane of the pulse $g(t)$ cannot be found $[13,26]$. In this work, the TF distribution of the pulse power has been calculated by means of the spectrogram obtained by application of the short-term-Fourier-transform [13], i.e. $\Theta_{l, m}=|\operatorname{STFT}(g(t))|^{2}$. Once the new definition of the SINR is provided, the MFP can be applied.

\subsection{Second Proposal}

In the second approach we consider the fact that in the case of GMC the key roles are played by the shapes of the transmit and receive pulses as well as by the distances between them; these shapes define the TF characteristics as well as the level of overlapping between neighboring pulses in TF plane. The main goal is to derive the definition of the equivalent channel model, as illustrated in Fig. 2.

In the ideal case the middle term of the formula (4), corresponding to the residual interference, is equal to zero, and the desired signal component, defined as $h_{l, m} d_{l, m}$, is maximal. However, due to the implementation impairment (i.e. inaccuracy in signal representation, finite duration of the transmit and receive filer, limited order of the filter etc.) some residual errors will be present in the received signal. In such a case formula (4) can be further rewritten as in (18):

$$
\begin{aligned}
\tilde{d}_{l, m} & =\tilde{h}_{l, m} p_{l, m} d_{l, m}+\tilde{I}_{l, m}+\sum_{n} \eta_{l, m}[n] \cdot q_{l, m}^{*}[n] \\
& =\tilde{h}_{l, m} p_{l, m} d_{l, m}+\hat{\eta}_{l, m} .
\end{aligned}
$$

In such a case the equivalent channel gain can be calculated as $\tilde{h}_{l, m}=h_{l, m} \cdot \Delta \epsilon_{l, m}^{(l, m)}$. The second term $\tilde{I}_{l, m}=\sum_{\left(l^{\prime}, m^{\prime}\right) \in \mathbb{D}_{l, m}} h_{l^{\prime}, m^{\prime}} d_{l^{\prime}, m^{\prime}} p_{l^{\prime}, m^{\prime}} \Delta \epsilon_{l^{\prime}, m^{\prime}}^{(l, m)}$ in (18) represents the total amount of interference introduced from the surrounding pulses to the pulse localized on the $(l, m)$ point on the time-frequency plane. Finally, the equivalent noise is now defined as $\hat{\eta}_{l, m}=\tilde{I}_{l, m}+\sum_{n} \eta_{l, m}[n] \cdot q_{l, m}^{*}[n]$. The above relation can be represented in the matrix form: 


$$
\tilde{\mathbf{D}}=\hat{\mathbf{H}} \mathbf{P D}+\tilde{\eta} .
$$

where

$$
\mathbf{D}=\left(d_{0,0} d_{0,1} \ldots d_{l, m} \ldots d_{L-1, M-1}\right)^{T}
$$

and

$$
\hat{\mathbf{D}}=\left(\hat{d}_{0,0} \hat{d}_{0,1} \ldots \hat{d}_{l, m} \ldots \hat{d}_{L-1, M-1}\right)^{T}
$$

are the column vector of the input data and their estimates, respectively, of the length $M \cdot L$. Next, $\mathbf{P}$ is the diagonal matrix filled with the values of allocated power, i.e. the diagonal of this matrix is defined as $\operatorname{diag}\left(p_{0,0} p_{0,1} \ldots p_{l, m} \ldots p_{L-1, M-1}\right) . \tilde{\eta}$ is the column matrix of the length $M \cdot L$ with the colored samples of noise, and $\hat{\mathbf{H}}$ is the $[M \cdot L \times M \cdot L]$ matrix containing the coefficient of the equivalent channel and is defined as in (22), where $\hat{h}_{l^{\prime}, m^{\prime}}^{(l, m)}=h_{l^{\prime}, m^{\prime}} \cdot \Delta \epsilon_{l^{\prime}, m^{\prime}}^{(l, m)}$. Due to the matrix representation, the matrix solutions derived for origin MFP $[17,20]$ can be applied.

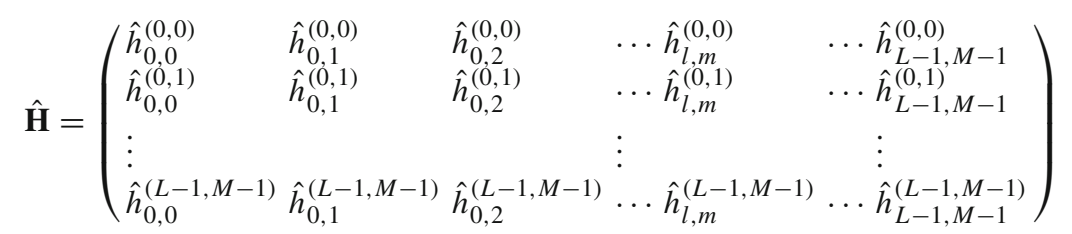

The main conclusion that can be drawn from such approximation is that the residual interference term is small thus can be treated as noise. In such a case the MFP can be applied in the straight-forward manner, although adapted to the two-dimensional case and taking into account the fact of non-ideal filter deign. Assuming the lack of dependency between the transit data $d_{l, m}$, the corresponding SINR $\gamma_{l, m}$ can be then calculated as:

$$
\gamma_{l, m}=\frac{\left|h_{l, m}\right|^{2} p_{l, m}\left|\Delta \epsilon_{l, m}^{(l, m)}\right|^{2}}{\sum_{\left(l^{\prime}, m^{\prime}\right) \in \mathbb{D}_{l, m}}\left|h_{l^{\prime}, m^{\prime}}\right|^{2} p_{l^{\prime}, m^{\prime}}\left|\Delta \epsilon_{l^{\prime}, m^{\prime}}^{(l, m)}\right|^{2}+\sigma^{2}\left|\Delta \epsilon_{l, m}^{(l, m)}\right|^{2}}
$$

\section{Simulation Results}

In order to verify the correctness of the presented analysis extensive computer simulations have been carried out. For both approaches described in the previous section the following setup has been defined. The size of one GMC frame equals $L \times M=16 \times 32$, the Prinz algorithm [25] has been applied for derivation of the dual pulse and the STFT-based spectrogram has been used for calculation of the power distribution on the TF plane. Gaussian pulse has been selected for transmission. Presented results have been obtained for the specific channel realization, which normalized transfer function is presented in Fig. 3. Furthermore, the pulse duration was equal to 1,024 samples, and it has been assumed that consecutive pulses are shifted by 896 samples (i.e. two pulses overlap over 128 samples).

The next three figures illustrate power allocation on TF plane obtained by application MFP and WFP for the Proposal II (described in Sect. 3.3). Figure4 shows the power allocation for the MFP when the transmission of BPSK symbols has been assumed, while Fig. 5 shows, how the power is allocated after application of the MFP when the input were Gaussian. Such results are of course in line with the origin results provided in [17-20] and show how the knowledge about the transmit signal distribution can improve the performance of the system. 


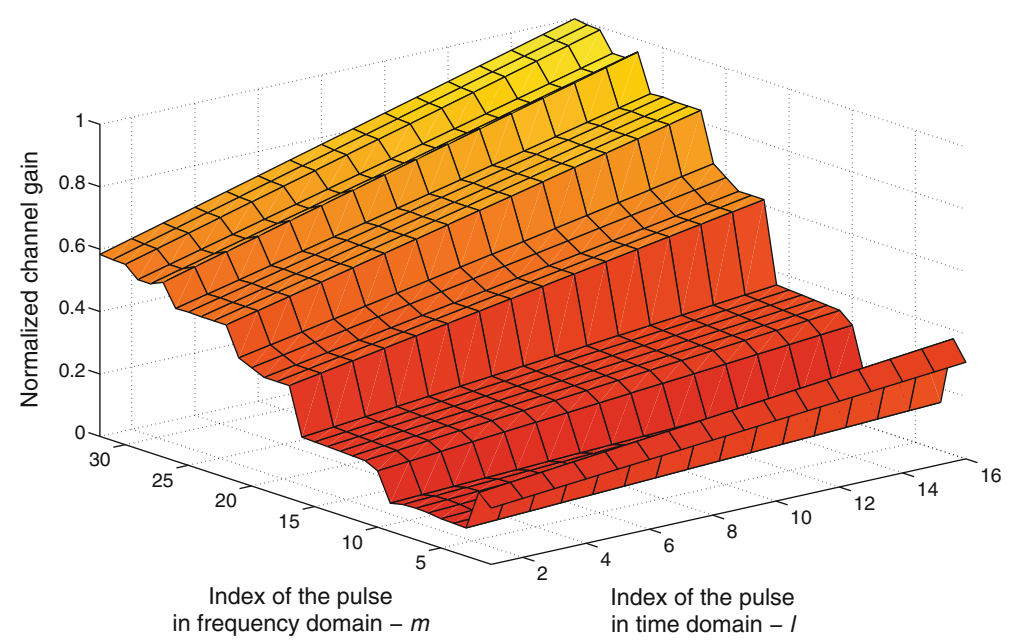

Fig. 3 Normalized channel transfer function

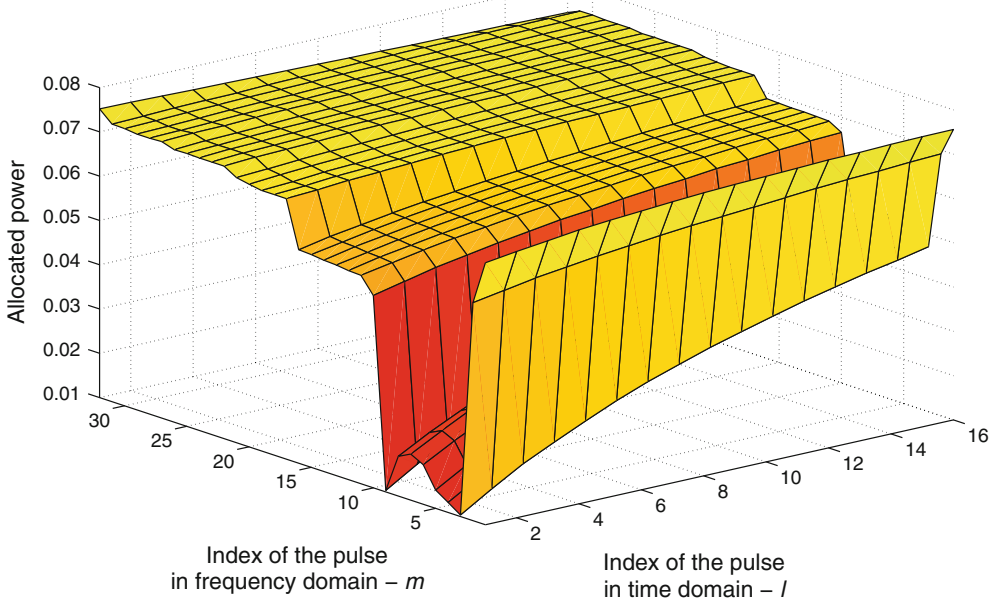

Fig. 4 Power allocation obtained for MFP, BPSK input; Gaussian pulses

This performance can be expressed either in the maximum achievable rate or minimum required total transmit power. Knowing that each pulse transmits one BPSK symbol, one can conclude that the increase of rate is significant. Furthermore, Fig. 6 shows how the power will be allocated when the WFP is applied. It can be stated that WFP and MFP with Gaussian inputs behave almost in the same manner. These observations, however, only prove the conclusions presented in $[17,18,20]$. The key observation is that the MFP has been applied for the GMC frame where overlapping pulses have been used. It is observable in all of the already mentioned figures on the edges of the frame. Clearly, the pulses located on the frame boarders will suffer from weaker interference thus the corresponding SINR $\gamma_{l, m}$ will be better.

Moreover, in order to illustrate the influence of the presence of residual interference, two separate figures have been presented, i.e. Figs. 7 and 8. Both figures represent the illustration of the MFP, however in the former the overlapping between pulses have been not considered. 


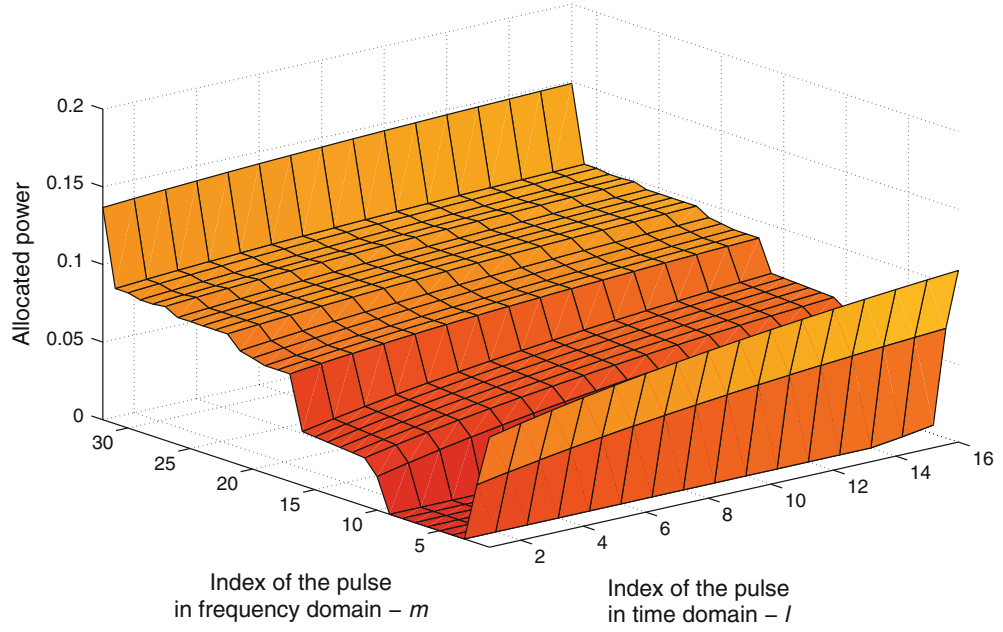

Fig. 5 Power allocation obtained for MFP, Gaussian input; Gaussian pulses

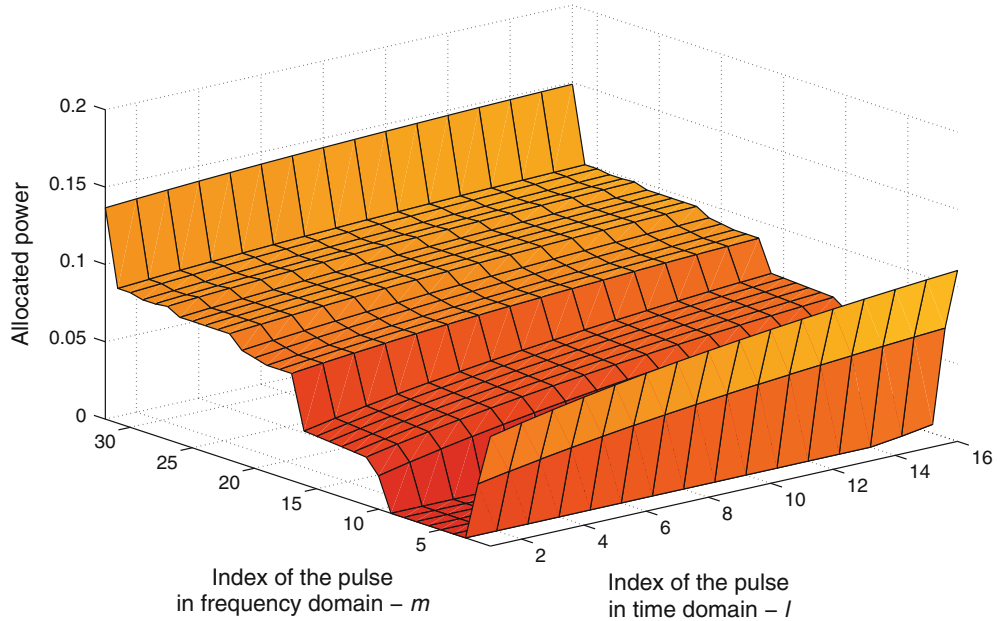

Fig. 6 Power allocation obtained for WFP; Gaussian pulses

In these figures three surfaces have been showed, among which the upper one represents the achieved water-level, while the surface at the bottom presents the inverse of the set of SINRs. In classical WFP one has to fill in the space between such two surfaces with water, what corresponds to the amount of power that has to be allocated for each pulse. Here, following the way provided in $[17,18,20]$, the middle surface is derived in the first step. Next, one has to fill the space (denoted in the figures by the letter B) with mercury, while the remaining space (denoted by capital A) with water. Again, the amount of water corresponds to the amount of power that has to be assigned to particular pulse. Comparison of these two figures shows, how the overlapping phenomena and the impairments in pulse design influence the final power allocation.

It is also worth showing how the power is allocated in one time slot over the whole transmission band-see Fig. 9. One can observe that MFP allocates power also for the pulses 


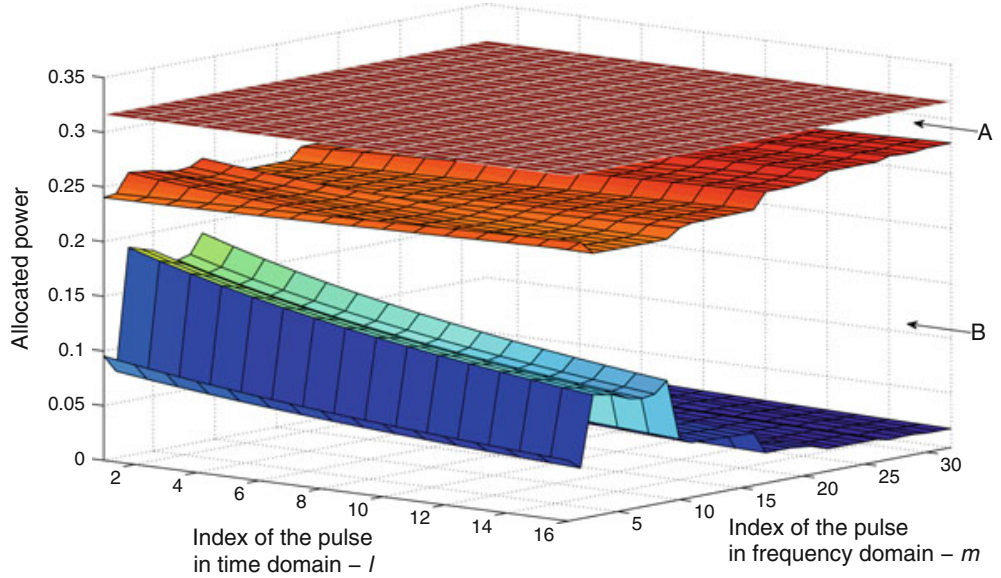

Fig. 7 Illustration of the Mercury-Water Filling principle for one GMC frame, overlapping between pulses is not included-straight forward approach

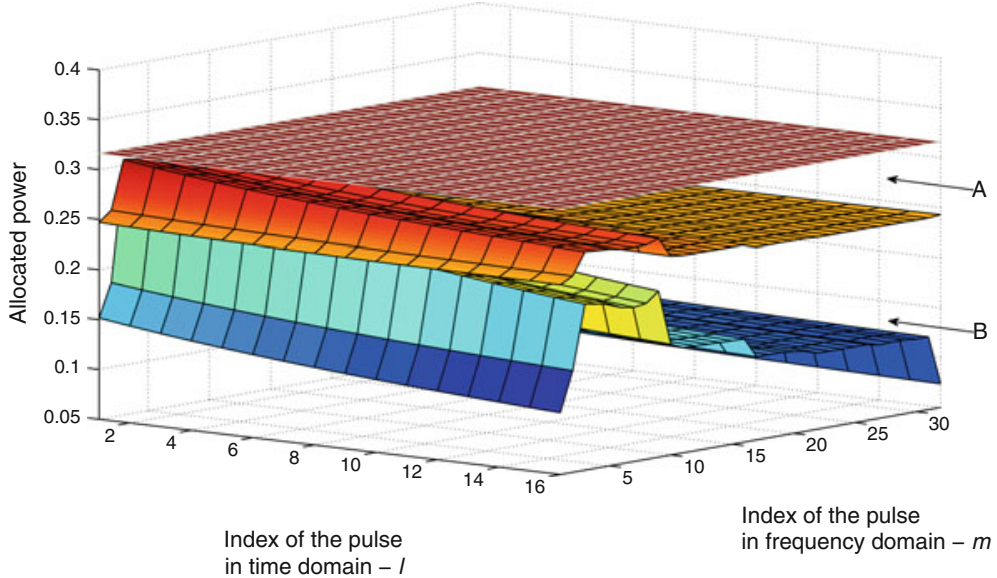

Fig. 8 Illustration of the MFP and WFP for one time slot in one GMC frame; overlapping between pulses is included

that are characterized by small SINR values. This is feasible since the MFP benefits from the knowledge about the input signal distribution. In that figure three cases have been shown, i.e. power allocation according to the MFP with BPSK inputs (solid line), and power allocation according to the WFP and MFP with Gaussian inputs. The two latter lines (both dashed) almost ideally overlap.

Finally, the influence of the pulse shape on the behavior of the MFP and WFP algorithm has been illustrated in Fig. 10. It can be noticed that the pulse shape and the distances between the pulses on TF plane has significant impact on the final power allocation, thus this phenomenon cannot be omitted.

Now, let us compare, how the power allocation will be realized when the first approach (described in Sect. 3.2) is considered. In that situation we do not include the impairments of the pulse design, but focus on the transmit side and allocate the power taking into account only 


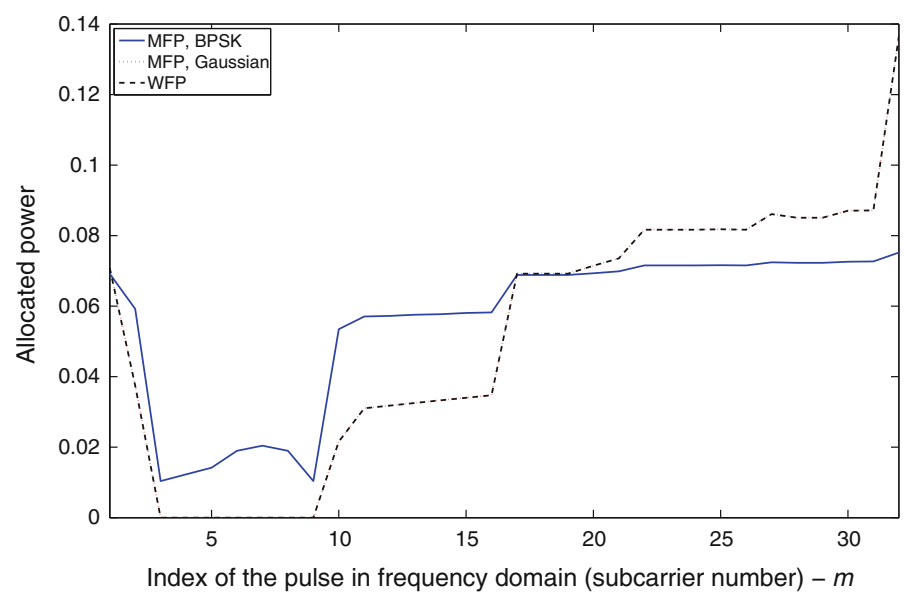

Fig. 9 Illustration of the MFP and WFP for one subcarrier in one GMC frame

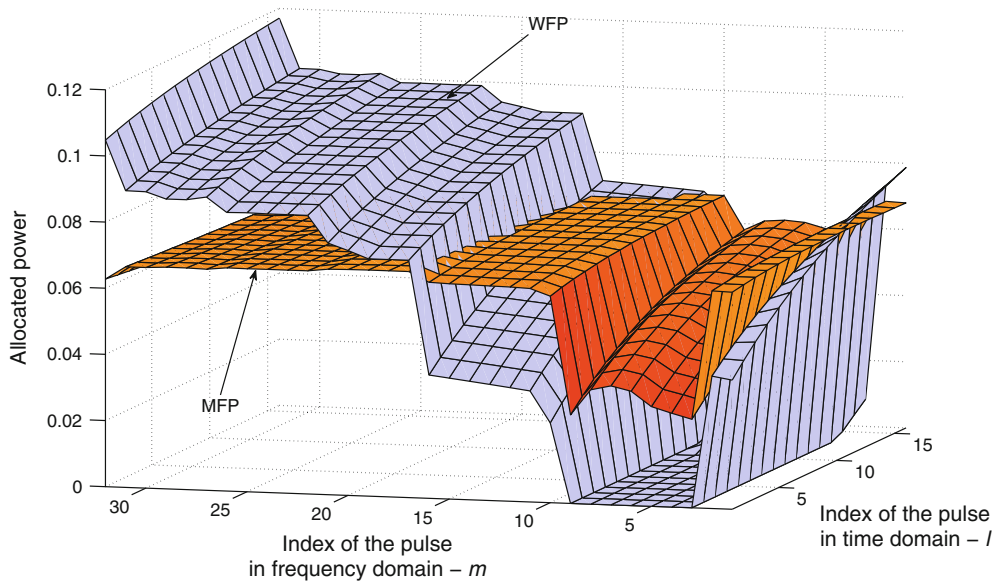

Fig. 10 Power allocation obtained for MFP and WFP for Kaiser pulse

the fact that the neighboring pulses overlap. One can notice that the final power allocation strongly depends on the duration of overlapping parts of two consecutive pulses. Figures 11 and 12 show the power allocation when the duration of the overlapping parts was equal to $12 \%$ and close to $0 \%$ of the pulse duration, respectively. Moreover, Fig. 13 presents the power allocation when the WFP has been applied. One can wonder, why such great amount of power has been allocated for the first and last column of pulses (i.e. for the time indexes equal 1 and 32). This is mainly due to the fact that although the original channel realization was defined as in Fig. 3, the influence of the overlapping pulses modifies significantly the final SINR values, as shown in Fig. 14. 


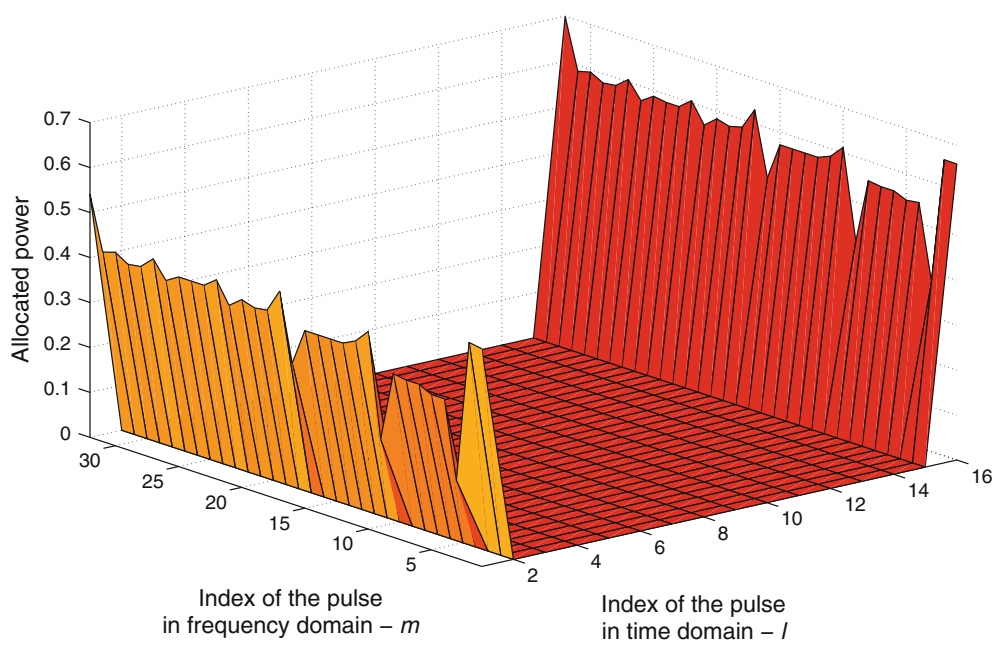

Fig. 11 Power allocation for MFP, strong overlapping

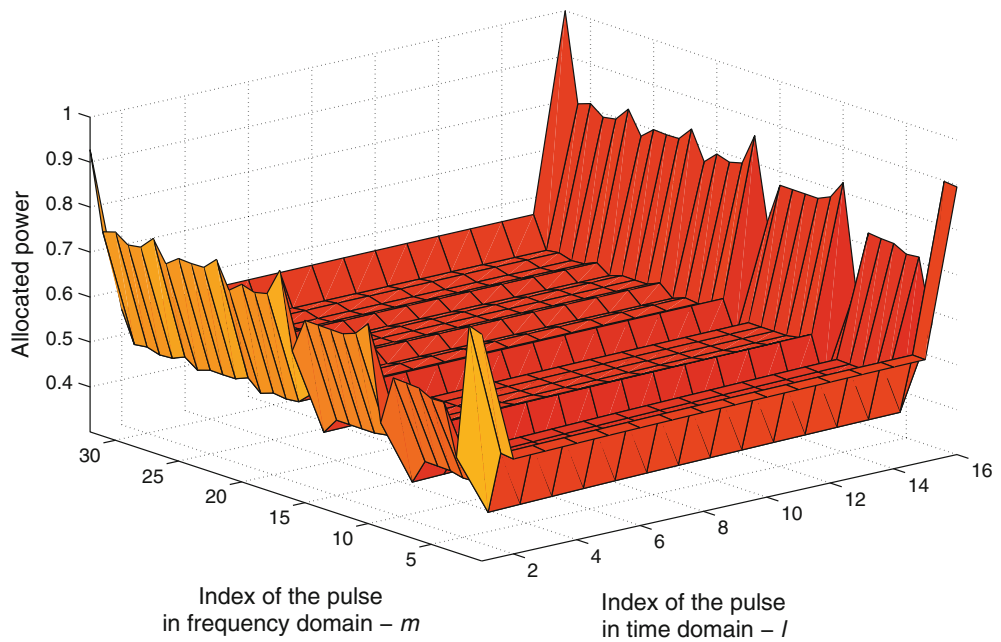

Fig. 12 Power allocation for MFP, weak overlapping

\section{Conclusions}

In the paper the analysis of the application of the mercury-waterfilling principle for the GMC signaling has been described. Two separate approaches have been proposed and tested by means of computer simulations. Obtained results have shown that inclusion of the overlapping phenomena (as in Sect. 3.2) influences significantly the final power allocation. The MFP, but also WFP, are sensitive to this phenomenon, since the stronger the overlapping, the stronger impact of the neighboring pulses on the SINR values. In the second approach, the equivalent channel model has been proposed and the new values of SINR have been calculated taking into account the presence of the residual interference. Based on the achieved results it has 


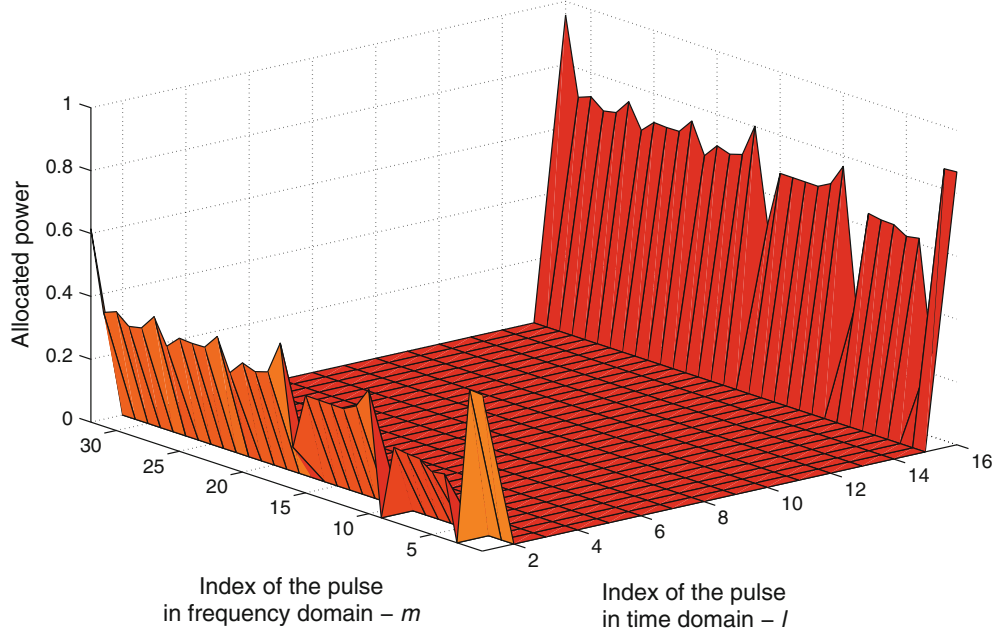

Fig. 13 Power allocation for WFP, strong overlapping

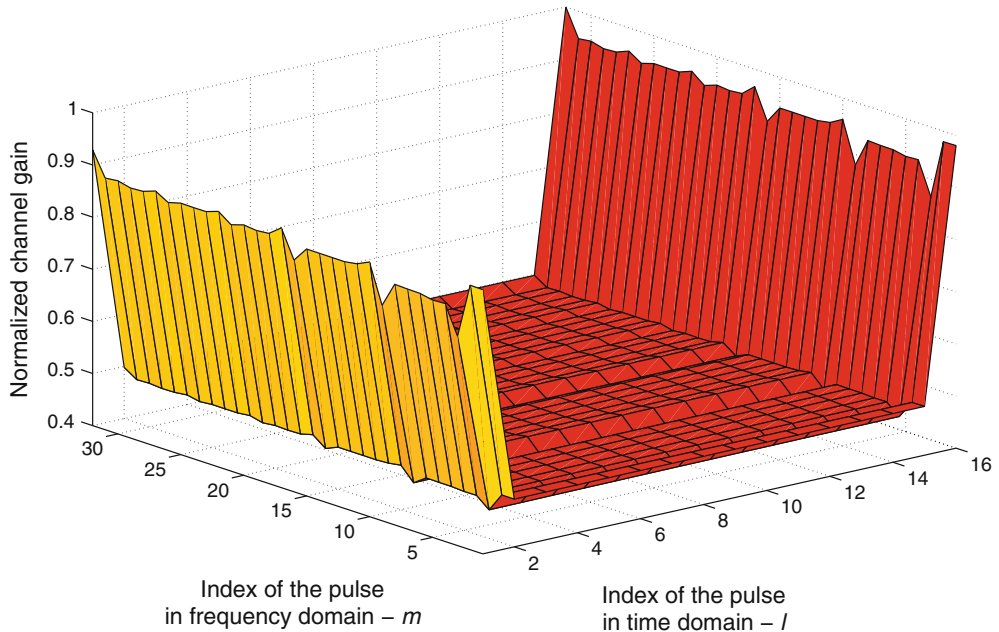

Fig. 14 SINR for one GMC frame when treating interference as noise, strong overlapping

to be stated that both approaches proved the necessity of consideration of GMC-specific features in the MFP algorithm.

Acknowledgments This work was supported by COST Action IC0902_ “Cognitive Radio and Networking for Cooperative Coexistence of Heterogeneous Wireless Networks". The author would like to thank Dr. Faouzi Bader for his valuable comments and suggestions.

Open Access This article is distributed under the terms of the Creative Commons Attribution License which permits any use, distribution, and reproduction in any medium, provided the original author(s) and the source are credited. 


\section{References}

1. IEEE Std 802.11n 2009. (2009, October) IEEE standard for information technology telecommunications and information exchange between systems. Local and metropolitan area networks. Specific requirements Part 11: wireless LAN medium access control (MAC) and physical layer (PHY) specifications amendment 5: enhancement for higher throughputs.

2. IEEE Std 802.16-2009. (2009, February). IEEE standard for local and metropolitan area networks Part 16: air interface for broadband wireless access systems.

3. ETSI EN 302755 V1.1.1 (2009-09). (2009, September). ETSI digital video broadcasting (DVB); Frame structure channel coding and modulation for a second generation digital terrestrial television broadcasting, system (DVB-T2).

4. Bingham, J. A. C. (1990). Multicarrier modulation for data transmission: An idea whose time has come. IEEE Communications Magazine, 28(5), 5-14.

5. Scaglione, A., Giannakis, G. B., \& Barbarossa, S. (1999). Redundant filterbank precoders and equalizers I. Unification and optimal designs. IEEE Transactions on Signal Processing, 47(7), 1988-2006.

6. Amini, P., \& Farhang-Boroujeny, B. (2009). Per-tone equalizer design and analysis of filtered multitone communication systems over time-varying frequency-selective channels. In IEEE international conference on communications, ICC'09 2009, 14-18 June (pp. 1-5).

7. Siohan, P., \& LaCaille, N. (1999). Analysis of OFDM/OQAM systems based on the filterbank theory. Global telecommunications conference, 1999. GLOBECOM '99 (Vol. 4, pp. 2279-2284).

8. EC-funded Project PHYDYAS. Physical layer for dynamic spectrum access and cognitive radio. http:// www.ict-phydyas.org/. Project lifetime 01.01.2008-31.10.2010. Accessed 06 March 2013.

9. EC-founded Project QoSMOS. Quality of service and MObility driven cognitive radio systems. http:// www.ict-qosmos.eu. Project lifetime 01.01.2010-31.12.2013. Accessed 06 March 2013.

10. Bellanger, M., Bonnerot, G., \& Coudreuse, M. (1976, April). Digital filtering by polyphase network: Application to sample rate alternation and filter banks. In Proceedings of IEEE transactions on acoustics, speech and signal processing (ICASSP'76) (Vol. 2, no. 2, pp. 109-114).

11. Du, J., \& Signell, S. (2007, February). Classic OFDM systems and pulse shaping OFDM/OQAM systems. TRITA-ICT/ECS R 07:01, ISSN 1653-7238.

12. Werner, Kozek, \& Molish, Andreas F. (1998). Nonorthogonal pulseshapes for multicarrier communications in Doubly dispersive channel. IEEE Journal on Selected Areas in Communications, 16(8), 1579-1589.

13. Feichtinger, H., \& Strohmer, T. (1998). Gabor analysis and algorithms. Theory and applications. Berlin: Birkhuser.

14. Siohan, P., \& Roche, C. (2000). Cosine-modulated filterbanks on extended Gaussian functions. IEEE Transactions on Signal Processing, 48(11), 3052-3061.

15. Bogucka, H., Wyglinski, A. M., Pagadarai, S., \& Kliks, A. (2011). Spectrally agile multicarrier waveforms for opportunistic wireless access. IEEE Communications Magazine, 49(6), 108-115.

16. Kliks, A., Stupia, I., Lottici, V., Giannetti, F., \& Bader, F. (2011, May). Generalized multi-carrier: An efficient platform for cognitive wireless applications. In 8th International workshop on multicarrier systems \& solutions (MC-SS'2011). Germany: Herrsching.

17. Guo, D., Shamai, S., \& Verdú, S. (2005). Mutual information and minimum mean-square error in Gaussian channels. IEEE Transactions on Information Theory, 51(4), 1261-1283.

18. Lozano, A., Tulino, A. M., \& Verdú, S. (2005, September). Mercury/waterfilling: Optimum power allocation with arbitrary input constellations. In Proceedings of IEEE international symposium on information theory, ISIT'05.

19. He, G., Gault, S., Debbah, M., \& Altman, E. (2008). Iterative mercury/waterfilling for parallel multiple access channels. In Proceedings of IEEE international conference on communications, ICC'08, China.

20. Perez-Cruz, F., Rodrigues, M. R. D., \& Verdu, S. (2010). MIMO Gaussian channels with arbitrary inputs: Optimal precoding and power allocation. IEEE Transactions on Information Theory, 56(3), 1070-1084.

21. Goldsmith, A. (2005). Wireless communication. Cambridge: Cambridge University Press.

22. Qian, S., \& Chen, D. (1999, March) Joint time-frequency analysis. IEEE Signal Processing Magazine, 16(2), 52-67. doi:10.1109/79.752051.

23. Matz, G., Schafhuber, D., Grochenig, K., Hartmann, M., \& Hlawatsch, F. (2007). Analysis, optimization, and implementation of low-interference wireless multicarrier systems. IEEE Transactions on Wireless Communications, 6(5), 1921-1931.

24. Vaidyanathan, P. P. (1993). Multirate systems and filters banks. Englewood Cliffs: PTR Prentice-Hall.

25. Prinz, P. (1996). Calculating the dual Gabor window for general sampling sets. IEEE Transactions on Signal Processing, 44(8), 2078-2082. 
26. Qian, S., \& Chen, D. (1993). Discrete Gabor transform. IEEE Transactions on Signal Processing, 41(7), 2429-2438.

27. Subbanna, N. K., \& Eldar, Y. C. (2004, September). A fast algorithm for calculating the dual Gabor window with integer oversampling. Proceedings. $23^{\text {rd }}$ IEEE Convention of Electrical and Electronics Engineers in Israel, 368-371. doi:10.1109/EEEI.2004.1361168.

28. Jung, P., \& Wunder, G. (2007). The WSSUS pulse design problem in multicarrier transmission. IEEE Transactions on Communications, 55(10), 1918-1928.

29. Ju, Z. (2010, March). A filter bank based reconfigurable receiver architecture for universal wireless communications. PhD dissertation, University of Kassel.

30. Hughes-Hartogs, D. Ensemble modem structure for imperfect transmission media. U.S. Patents No. 4679227 July 1987, 4731816 March 1988 and 4833796 May 1989.

31. Campello, J. (1999). Practical bit loading for DMT. In IEEE international conference on communications, ICC'99 (Vol. 2, pp. 801-805).

32. Fischer, R. F. H., \& Huber, J. B. (1996). A new loading algorithm for discrete multitone transmission. In Global telecommunications conference, 1996. GLOBECOM 96. Communications: The key to global prosperity, 18-22 (Vol. 1, pp. 724-728).

\section{Author Biography}

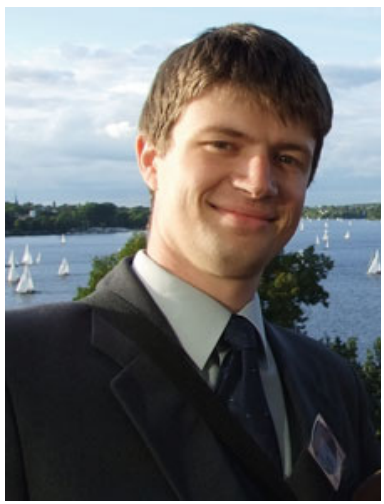

Adrian Kliks received his M.Sc. and Ph.D. degrees in Telecommunication from Poznan University of Technology in 2005 and 2011, respectively. Since 2005 he has been employed at the Institute of Electronics and Telecommunications, and since 2007 as the senior researcher at the Chair of Wireless Communication in the Faculty of Electronics and Telecommunication. Starting from October 2011 he works as assistant professor at Poznan University of Technology. Since 2012 he is IEEE Senior Member, and is an author or coauthor of around 40 scientific papers. His research interests cover the wide spectrum of wireless communications, focusing on the multicarrier (both orthogonal an non-orthogonal) systems, cognitive and software radio including standardization activities, as well as in hardware implementation and measurements, as well as in radio-planning. 